\title{
Respiratory Effects of Hexachlorocyclopentadiene on Intact Rainbow Trout (Salmo gairdneri) and on Oxidative Phosphorylation of Isolated Trout Heart Mitochondria
}

\author{
Palarp Sinhaseni, ${ }^{* 11}$ Louis G. D'Alecy, $\dagger$ Rolf HaRtung, ${ }^{*}$ \\ AND Marshal Shlafer $\ddagger^{2}$
}

\begin{abstract}
*Institute of Environmental and Industrial Health, †Department of Physiology, and $\ddagger$ Department of Pharmacology, University of Michigan Medical School, Ann Arbor, Michigan 48109
\end{abstract}

Received August 11, 1982; accepted October 4, 1982

\begin{abstract}
Respiratory Effects of Hexachlorocyclopentadiene on Intact Rainbow Trout (Salmo gairdneri) and on Oxidative Phosphorylation of Isolated Trout Heart Mitochondria. SinhasenI, P., D'Alecy, L. G., Hartung, R., AND Shlafer, M. (1983). Toxicol. Appl. Pharmacol. 67, 215223. Acclimated normal rainbow trout were exposed to $130 \mathrm{ppb}$ hexachlorocyclopentadiene (HEX) in a flow-through well water circuit which was designed to permit measurements of oxygen consumption by the fish. Compared to preHEX values, HEX increased oxygen consumption rates by $186 \pm 24 \%(\bar{x} \pm$ SEM), with maximum oxygen consumption rates being reached in approximately $84 \mathrm{~min}$ after HEX exposure. Oxygen consumption subsequently decreased, and all HEX-exposed fish died within $6.5 \mathrm{hr}$ of exposure. Fish exposed to HEX-free vehicle (acetone) showed no changes of oxygen consumption. When added to normal isolated trout heart mitochondria, HEX appeared to uncouple oxidative phosphorylation, with calculated respiratory control ratios being decreased $50 \%$ from control values at a HEX concentration of $0.41 \mu \mathrm{M}$. We postulate that one important mechanism of HEX intoxication in the intact animal may be due to increased oxygen consumption and impaired oxidative ATP synthesis due to the mitochondrial uncoupling action of the toxicant.
\end{abstract}

Hexachlorocyclopentadiene (C-56; HCCPD; HCPD; HEX) is a highly reactive and toxic conjugated diene. An estimated $2.3 \times 10^{7} \mathrm{~kg}$ of HEX are produced annually ( $\mathrm{Lu}$ et al., 1975) for use in the manufacture of various cyclodiene insecticides and of flame retardants synthesized from chlorendic acid and anhydride. The chemical poses a potential industrial and environmental hazard, and accidental poisoning of humans and other animals has been reported (Kominsky et al., 1980; Lawrence and Dorough, 1981; Spehar et al., 1979). Nevertheless, although the hu-

\footnotetext{
${ }^{1}$ This work was conducted in partial fulfillment of the requirements for the degree of Doctor of Philosophy (Toxicology).

${ }^{2}$ To whom all correspondence should be addressed.
}

man toxicity has been described (Kominsky et al., 1980) and animal studies of HEX intoxication have been published (Patty, 1963; Treon et al., 1955; Spehar et al., 1979), little is known about the mechanisms by which the agent produces its deleterious actions.

In this paper we report that HEX produces profound respiratory stimulation, followed by death, of intact rainbow trout (Salmo gairdneri) exposed to the toxicant in a controllcd, flow-through system. We also report preliminary data showing that HEX acts as a potent uncoupler of mitochondrial oxidative phosphorylation, and suggest that this cellular action may constitute one of the important mechanisms by which HEX toxicity to the whole organism may be occurring. 


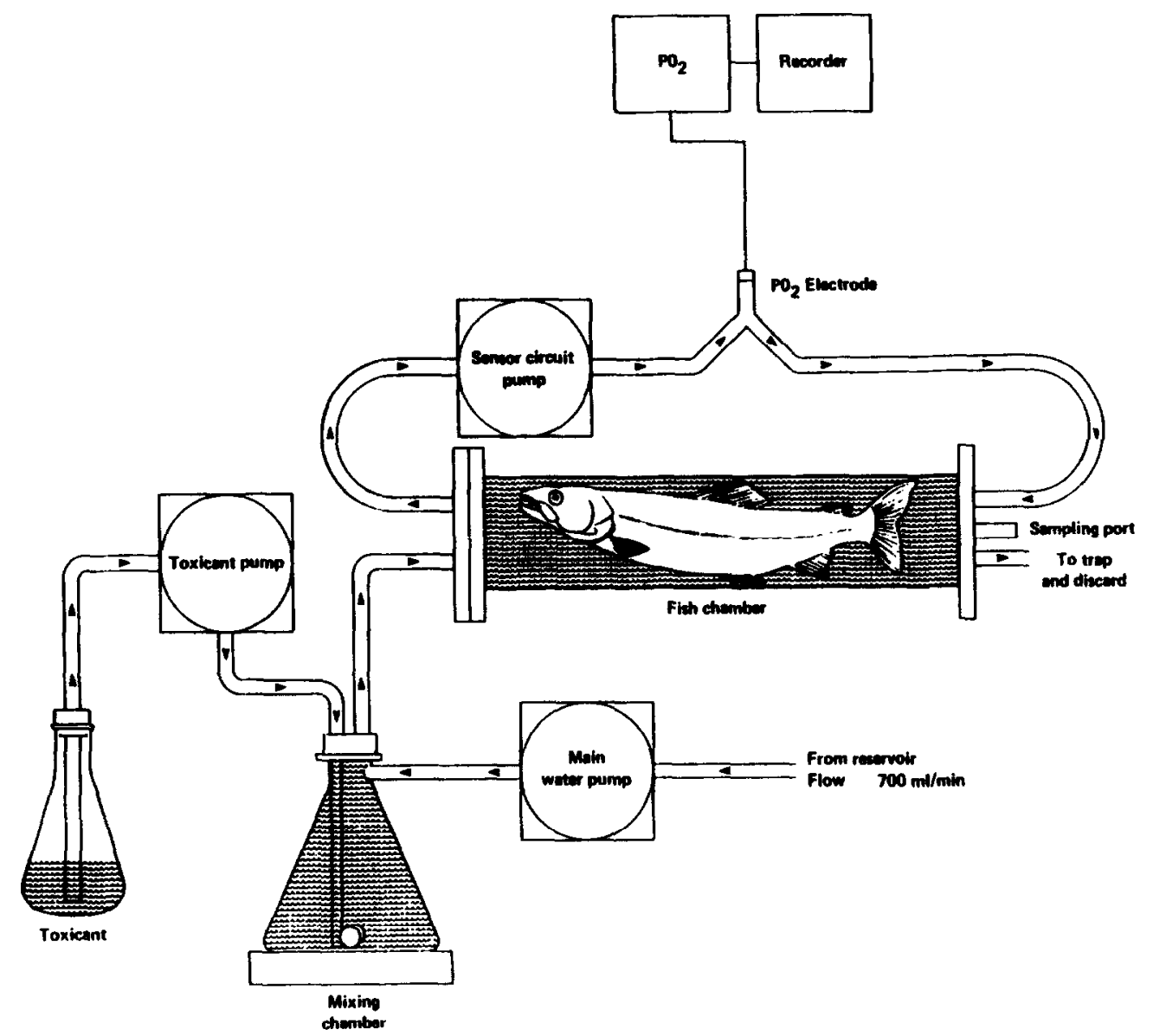

Fig. 1. Two pumps were used to deliver aerated well water and toxicant (final concentration $130 \mathrm{ppb}$ ) to the fish chamber. For measurements of oxygen consumption by the fish, a third pump was used to circulate water through the chamber and past an oxygen $\left(\mathrm{PO}_{2}\right)$ electrode, and flow from the other pumps was temporarily stopped so that the sensor circuit was isolated. Opercular and coughing rates were counted by observing the fish in the clear plastic chamber. The entire system was kept at $12^{\circ} \mathrm{C}$.

\section{METHODS}

Hexachlorocyclopentadiene. HEX, purchased from Aldrich Chemical Company (Milwaukee, Wisc.), was purified by vacuum distillation. Purity of the distilled HEX was $98.6 \%$ based on gas-liquid chromatography and mass spectrometry. For each experiment a stock solution of 12 $\mathrm{mM}$ was prepared daily in acetone. For the mitochondrial studies described below, other stock solutions in ethanol were prepared by dilution of the $12 \mathrm{mM}$ solution so that HEX concentration, rather than the volume of the diluent, was the major experimental variable.

Trout. Rainbow trout (Salmo gairdneri; average body weight approximately $268 \mathrm{~g}$ ) were obtained from the Michigan Department of Natural Resources (Oden Hatchery Station, Oden, Mich.). The fish were acclimated in a flow-through well water system at the Great Lakes Fishery Laboratory (Ann Arbor, Mich.) for at least 2 weeks before their use in the experiments.

Intact trout exposure system. Figure 1 shows a schematic drawing of the exposure system. The major components were a main water supply, a HEX administration circuit, and a sensor circuit used for measuring oxygen consumption. The entire system, plus the water used, was maintained at $12^{\circ} \mathrm{C}$.

The main water pump delivered vigorously aerated well water at a rate of $700 \mathrm{ml} / \mathrm{min}$ through a mixing chamber, the fish chamber, and finally through activated charcoal filters which removed the toxicant before discharge. The mixing chamber contained HEX which was delivered by a precision pump to the main circuit at a rate sufficient to give a concentration of 130 parts per billion (ppb). In other experiments, HEX-free acetone was the control. 
When oxygen consumption measurements were made, the main water and toxicant pumps were turned off, the main inflow and outflow paths to the fish chamber were closed, and the sensor pump was used to circulate water at a rate of $380 \mathrm{ml} / \mathrm{min}$ past the oxygen electrode. The concentration of HEX selected was chosen largely on the basis of preliminary data obtained in studies of the tissue distribution of radiolabeled HEX (Sinhaseni, 1982) which indicated that the 4-hr $\mathrm{LC}_{100}$ of HEX in a static exposure system was $120 \mathrm{ppb}$.

Total oxygen content of the water was calculated by the Bunsen coefficient, corrected for ambient temperature and barometric pressure. The oxygen electrode was calibrated with air- and nitrogen-equilibrated well water before and after each experiment. Care was taken not to allow oxygen content of the sensor circuit to fall below $5 \mathrm{ppm}$ during measurements of oxygen consumption to avoid producing hypoxia (Prosser, 1973). Measurement periods lasted between 2 and $15 \mathrm{~min}$, depending on the size of the fish and the actual oxygen consumption rates. Once measurements were completed, normal circulation of well water from the main pump was resumed.

Fish were acclimated in the circuit for $15 \mathrm{hr}$ before exposure to HEX. During the experiments, rates of opercular movement and of coughing were counted by an observer. Death was defined as a cessation of observable opercular movement for $1 \mathrm{~min}$ or more.

Studies with isolated trout mitochondria. Mitochondria were isolated from the hearts of trout which had been acclimated for at least 7 days in a flow-through well water system at the Great Lakes Fishery Laboratory. For each of the six experiments, 7 to 13 fish were quickly decapitated and their heart removed and placed in a beaker of ice-cold isolation medium $(180 \mathrm{mM} \mathrm{KCl}, 10 \mathrm{mM}$ TrisEDTA, and $0.5 \%(\mathrm{w} / \mathrm{v})$ bovine serum albumin, $\mathrm{pH} 7.40$, at $4{ }^{\circ} \mathrm{C}$ ). The solution was decanted to remove as much blood as possible, fresh medium was added, and the hearts were quickly blotted, weighed, and returned to the medium. Heart weight was $432 \pm 13 \mathrm{mg}$ ( $\bar{x} \pm$ SEM). Mitochondria were then isolated by differential centrifugation, exclusively in the medium described above, as described elsewhere (Shlafer et al., 1981). The final mitochondrial pellet was gently resuspended in a small, measured volume of fresh isolation medium. The protein concentration of the suspension was measured by the Biuret assay with bovine serum albumin as the protein standard. Mitochondrial respiratory activity was measured immediately, and all measurements were completed within $2 \mathrm{hr}$ of final isolation.

Mitochondrial oxygen consumption was measured polarographically in a Gilson Oxygraph equipped with a Clark electrode in a medium containing $0.22 \mathrm{M}$ sucrose and $4.4 \mathrm{mM} \mathrm{K}_{2} \mathrm{HPO}_{2}$ in $13 \mathrm{~mm}$ morpholinopropanesulfonic acid (MOPS; pH 7.40 at $30^{\circ} \mathrm{C}$ ). Mitochondrial protein concentration in the cuvette was $1.06 \pm 0.33 \mathrm{mg} / \mathrm{ml}$, and glutamate plus malate ( $7.5 \mathrm{mM}$ of each, Tris salts) was the substrate. The mitochondria were incubated in this medium, with or without HEX or its solvent $(20 \mu \mathrm{l}$ of absolute ethanol) for $2 \mathrm{~min}$, during which time basal (or initial State 4) respiratory rate was recorded. Five hundred nanomoles of $\mathrm{Na}_{2} \mathrm{ADP}$ (final concentration 310 $\mu \mathrm{M})$ was then added to induce State 3 respiration. In those experiments in which mitochondria returned to State 4 respiration after the first ADP addition (generally, these were the control experiments or those in which only very low HEX concentrations were used), a second 500-nmol aliquot of ADP was added. The sequence of testing the various concentrations of HEX or the controls (oxygen consumption in the absence of HEX or of HEX-free ethanol) was varied randomly. For each mitochondrial preparation, each concentration of HEX was tested at least in duplicate. Based on the estimated solubility of oxygen in a medium similar to that which we used, we calculated that the total volume of assay medium (1.605 $\mathrm{ml}$ ) contained $385 \mathrm{nmol} \mathrm{O}_{2}$, or 770 natom of oxygen, and this number was used as the basis for our calculations of mitochondrial oxygen consumption rates.

State 3 respiratory rate was defined as the rate of oxygen consumption (natom $\mathrm{O} / \mathrm{min} / \mathrm{mg}$ protein) in the presence of inorganic phosphate, substrate, and added ADP. State 4 respiratory rate was defined as that measured in the presence of phosphate and substrate, but in the absence of ADP or after its consumption. Respiratory control ratios were calculated as the quotient of the State 3 respiratory rate and the subsequent State 4 rate. When mitochondria failed to return to State 4 respiration after ADP addition (as when high HEX concentrations were tested), a respiratory control index was calculated as the State 3 rate and the previous (initial) State 4 rate. The ADP:O ratio was calculated as the quotient of the amount of ADP added ( $550 \mathrm{nmol}$ ) and the corresponding amount of oxygen consumed due to the ADP addition.

Statistical comparisons to evaluate the effects of HEX were made by paired $t$ tests. Most comparisons were made between values obtained with HEX and values obtained with the HEX-free, ethanol-treated controls.

\section{RESULTS}

The validity of the subsequent oxygen consumption measurements upon HEX exposure depends greatly upon the stability of the model under basal conditions (Skidmore, 1970). We therefore show in Fig. 2 oxygen consumption rates of six representative trout during acclimation in the flow-through chamber. Because of handling, initial oxygen consumption rates were expectedly high, but these stabilized in an average of $5.5 \mathrm{hr}$ and stayed relatively constant thereafter, provided no HEX was added. Fifteen hours after they were 


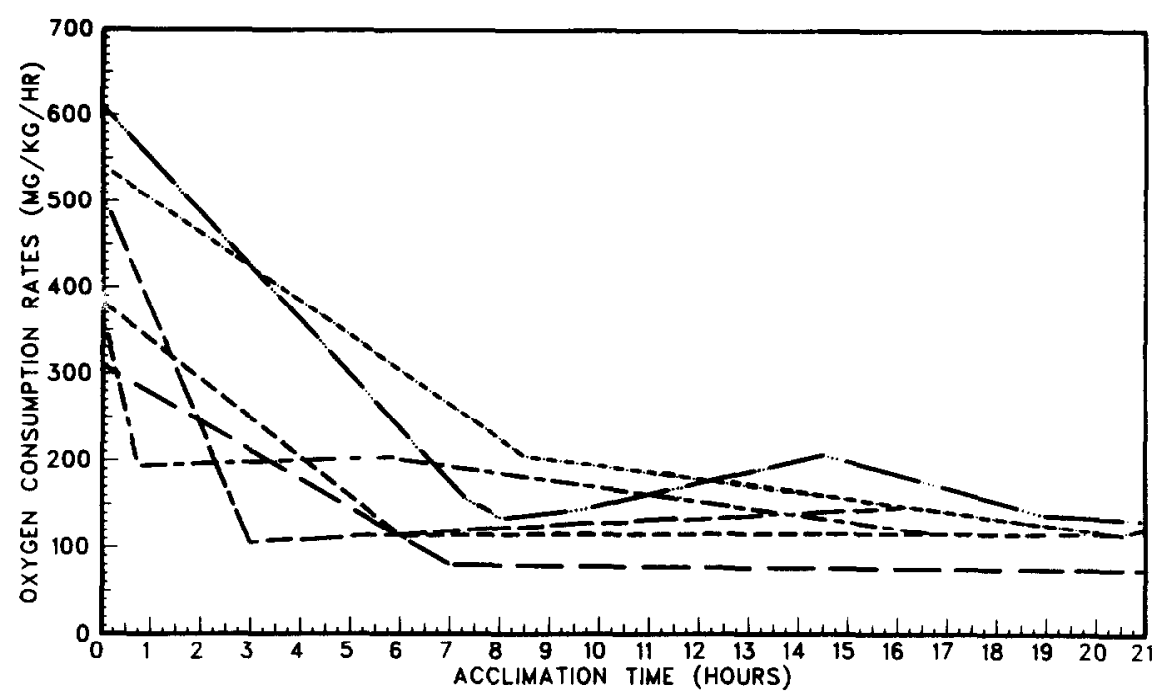

FIG. 2. Oxygen consumption rates of six different fish (each represented by a different dotted line) were initially high upon placing the fish into the flow-through apparatus, but ultimately stabilized, as long as HEX was not added.

placed in the chamber, the time at which HEX exposure was begun, the oxygen consumption rate of 13 fish which were exposed to HEX or to acetone was $141 \pm 13 \mathrm{mg} / \mathrm{kg} / \mathrm{hr}(\bar{x} \pm 1$ SEM), and if HEX were not added the rate changed little by the $21 \mathrm{st} \mathrm{hr}$, when observations and measurements were usually stopped (Fig. 3). These basal oxygen consumption values are within normal limits for this size and species of fish (Skidmore, 1970). During this

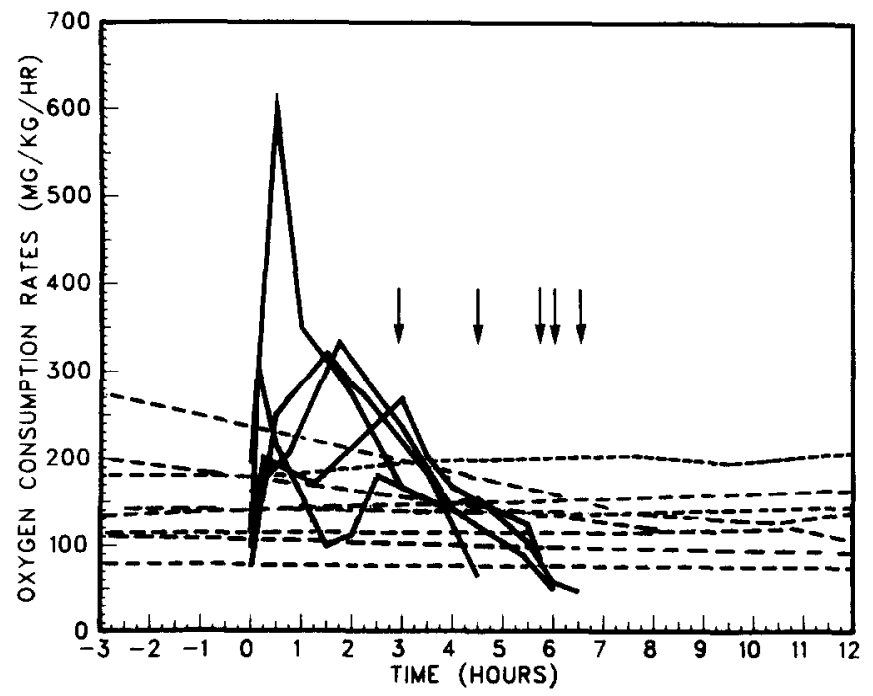

FIG. 3. Solid lines show increases of oxygen consumption rates by fish exposed to $130 \mathrm{ppb}$ HEX after equilibration. Dotted lines represent oxygen consumption rates of fish exposed to HEX-free acetone, and show no significant respiratory-stimulating effects of the solvent alone. Vertical arrows denote the time of death of the HEX-exposed fish. In contrast to the prolonged viability of control fish, all fish exposed to HEX died within $6.5 \mathrm{hr}$ of toxicant exposure. 
acclimation period opercular rates were relatively stable at approximately $80 / \mathrm{min}$, and coughing rates ranged from 0 to $4 / \mathrm{min}$.

Figure 3 shows the oxygen consumption rates of five fish exposed to $130 \mathrm{ppb}$ HEX, and of eight exposed to HEX-free acetone (147 ppm; controls). Acetone exposure produced no remarkable changes of oxygen consumption or opercular and coughing rates. However, oxygen consumption rates of the HEXexposed fish increased by $186 \pm 24 \%(\bar{x} \pm 1$ SEM) from the preexposure value. Upon reaching a peak after HEX exposure, oxygen consumption rates then declined with a variable time course, and within $6.5 \mathrm{hr}$ after exposure $(\bar{x} 5.2 \mathrm{hr}$ ), all HEX-exposed fish were dead. The first HEX-treated fish died $3 \mathrm{hr}$ after exposure (Fig. 3).

When expressed in terms of actual oxygen consumption rates, average maximum oxygen consumption of HEX-treated fish was 370 $\pm 64 \mathrm{mg} / \mathrm{kg} / \mathrm{hr}(p<0.01$ from the preexposure value of $132 \pm 22 \mathrm{mg} / \mathrm{kg} / \mathrm{hr}$, based on a paired $t$ test). The peak increase of oxygen consumption rate occurred in an average of $84 \pm 31$ min after HEX exposure. When measured at a comparable time, oxygen consumption by control fish was only $144 \pm 16$ $\mathrm{mg} / \mathrm{kg} / \mathrm{hr}$ (not significantly different from the preexposure value of $146 \pm 18 \mathrm{mg} / \mathrm{kg} / \mathrm{hr}$ ).

The data summarized in Table 1 reveal further information about the effects of HEX or acetone on oxygen consumption and survival. First, although oxygen consumption rates of HEX-exposed fish initially rose to a peak, falling thereafter, both oxygen consumption rates and total oxygen consumed (determined by planimetric analysis of oxygen consumption curves such as those shown in Fig. 3) remained significantly higher in HEX-exposed fish than in controls for at least $3 \mathrm{hr}$. As a group, total oxygen consumed by HEX-treated fish remained significantly greater than that of controls for up to $5.2 \mathrm{hr}$. Table 1 shows that $5.2 \mathrm{hr}$ after exposure (the average timeto-death), oxygen consumption rates of HEXexposed fish had dropped to $72 \pm 30 \mathrm{mg} / \mathrm{kg} /$ $\mathrm{hr}$, a value which was significantly lower than that of controls measured at this time. However, by $5.2 \mathrm{hr}$ the reduced oxygen consumption rates and total amount of oxygen consumed reflect the fact that all HEX-exposed fish had passed the time of maximal oxygen consumption, and that three of five fish had died. Although Table 1 reveals that the survival rate of HEX-exposed fish was significantly different from control only at $6.5 \mathrm{hr}$ of exposure, the lack of a significant difference at earlier times (e.g., $5.2 \mathrm{hr}$ ) is likely to be due to the small sample sizes which we studied.

In the HEX-exposed fish opercular movements and coughing rates were elevated, but unlike the transient changes of oxygen consumption rates, opercular and coughing rates remained high, at approximately 140 and $40 /$ $\mathrm{min}$, respectively, throughout HEX exposure. Opercular and coughing rates declined only immediately before death.

Light and scanning electron microscopy of gills from control and HEX-exposed fish revealed no significant changes except for moderate thickening of the lamellae of exposed fish, and this change was evident only upon electron microscopic examination.

Figure 4 shows typical oxygen consumption records of isolated normal trout heart mitochondria and those exposed to HEX-free solvent (ethanol) or to HEX at concentrations from 0.59 to $14.4 \mu \mathrm{M}$. Control mitochondria were tightly coupled (respiratory control ratio $8.7 \pm 0.6$; Table 2) and phosphorylated ADP efficiently (ADP:O ratio $3.04 \pm 0.12$; Table 2). Adding HEX-free ethanol $(20 \mu \mathrm{l}$ of absolute ethanol in a total cuvette volume of $1.605 \mathrm{ml})$ had no significant effect on mitochondrial performance and hence these ethanol-treated organelles were used as controls for subsequent comparison with values obtained from HEX-treated mitochondria.

The major effect of briefly incubating mitochondria with HEX was an increase of State 4 respiratory rates (Figs 4, 5), with no appreciable change of State 3 rates (Figs 4,5). This change resulted in a significant decrease of the calculated respiratory control ratios at HEX concentrations of $0.059 \mu \mathrm{M}$ or more (Table 
TABLE 1

OXYgen Consumption RATES (OCR), TOTAL OXYGen CONSUMPTION (TOC), AND Number OF FISH SURVIVING EXPOSURE TO HEX OR TO HEX-FrEe ACETONE ${ }^{a}$

\begin{tabular}{lrrr}
\hline & Control & HEX & $P^{b}$ \\
\hline OCR (mg/kg/hr) & & & n.s. \\
Preexposure & $146 \pm 18$ & $132 \pm 22$ & $<0.05$ \\
84 min postexposure ${ }^{b}$ & $144 \pm 16$ & $226 \pm 41$ & $<0.01$ \\
$3 \mathrm{hr}$ postexposure & $136 \pm 14$ & $219 \pm 22$ & $<0.001$ \\
$5.2 \mathrm{hr}$ postexposure & $132 \pm 16$ & $72 \pm 30$ & $<0.001$ \\
$6.5 \mathrm{hr}$ postexposure & $132 \pm 16$ & $0 \pm 0$ & $<0.01$ \\
TOC (mg/kg) & & & $<0.01$ \\
$84 \mathrm{~min}$ postexposure & $220 \pm 26$ & $398 \pm 59$ & $<0.05$ \\
$3 \mathrm{hr}$ postexposure & $438 \pm 50$ & $736 \pm 74$ & n.s. \\
$5.2 \mathrm{hr}$ postexposure & $735 \pm 80$ & $1043 \pm 67$ & n.s. \\
$6.5 \mathrm{hr}$ postexposure & $856 \pm 90$ & $1101 \pm 66$ & n.s. \\
Survivors $(n / n)$ & & & n.s. \\
$84 \mathrm{~min}$ postexposure & $8 / 8$ & $5 / 5$ & $<0.005$ \\
$3 \mathrm{hr}$ postexposure & $8 / 8$ & $4 / 5$ & $3 / 5$ \\
$6.5 \mathrm{hr}$ postexposure & $8 / 8$ & $0 / 5$ & \\
\hline
\end{tabular}

\footnotetext{
${ }^{a}$ Values are arithmetic $\bar{x} \pm 1$ SEM.

${ }^{b}$ Probabilities calculated by Student's $t$ test for OCR and TOC, and by Fisher's exact test for survival data (n.s., not significant).

${ }^{c}$ Eighty-four minutes was selected as the average time at which HEX-stimulated respiration was maximized in the five exposed fish. The average time of death of HEX-exposed fish was $5.2 \mathrm{hr}$. Six and one-half hours was the time at which all fish had died. Three hours was used as a measurement time which was intermediate between the first two postexposure measurements.
}

2). Compared to values calculated from ethanol-treated controls, respiratory control was decreased $50 \%$ by an average HEX concentration of $0.41 \mu \mathrm{M}$, which was calculated to be equivalent to $0.60 \mathrm{nmol}$ of $\mathrm{HEX} / \mathrm{mg}$ mitochondrial protein in the assay cuvette. The data also showed a trend indicative of decreasing ADP:O ratios with HEX concentrations of $0.15 \mu \mathrm{M}$ or more (Table 2), but the mitochondria exposed to HEX concentrations higher than this failed to return to State 4 respiration after adding the 500-nmol aliquot of ADP, and so the ADP:O ratio was not calculated for these concentrations.

\section{DISCUSSION}

Under experimental conditions HEX can produce sublethal toxicity or death when ad- ministered to animals by various routes of administration (Lawrence and Dorough, 1981; Mehendale, 1977; Patty, 1963; Spehar et al., 1979; Treon et al., 1955). Although the individual sequelae associated with HEX intoxication vary with the route of administration, the toxicant generally produces necrotizing lesions at sites of direct contact, and also diffuse degenerative lesions in most organs to which the parent compound or metabolites are delivered by the systemic circulation. Accidental exposure of HEX to humans has also occurred (Kominsky et al., 1980), producing a syndrome characterized by headache, dyspnea, hematological alterations indicative of hepatic dysfunction, and cutaneous, conjunctival, and respiratory tract irritation. Although the toxicity of HEX can be demonstrated readily in the laboratory, there is little definitive information about the fate of HEX 
in the environment or in the organisms exposed to it (Atallah et al., 1981; Lu et al., 1975; Veith et al., 1979), about the magnitude of the environmental risk which it poses, or about the mechanisms by which toxic manifestations to HEX exposure occur.

Our studies of the respiratory effects of HEX were prompted by earlier work which revealed pulmonary damage in terrestrial animals and gill damage in aquatic species exposed to HEX, and accumulation of $\left[{ }^{14} \mathrm{C}\right] \mathrm{HEX}$ in respiratory structures (Khan et al., 1981; Lawrence and Dorough, 1981; Sinhaseni, 1982). These observations suggested that a primary target of damage by HEX might be the respiratory tract, with toxicity being manifest as respiratory depression leading to hypoxia, possibly due to impaired gas transport into the systemic circulation, ultimately leading to the organism's death.

In contrast to the expected outcome, data obtained in the present study suggest a marked

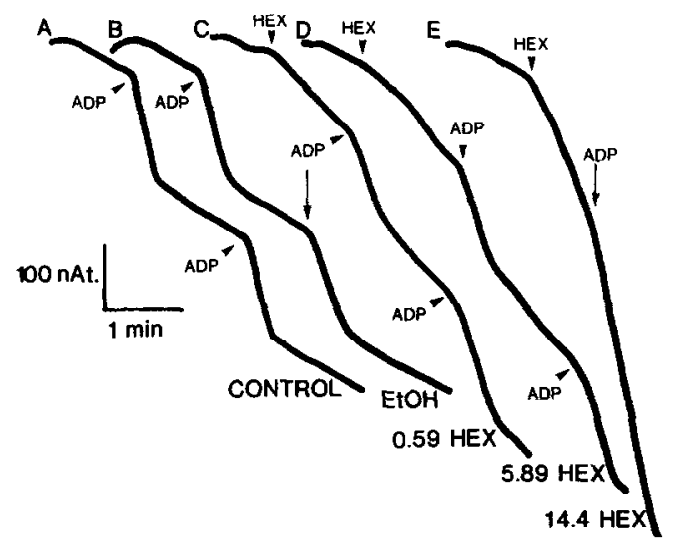

FIG. 4. Polarographic recordings of mitochondrial oxygen consumption show the effects of increasing concentrations of HEX ( $\mu \mathrm{mol} / \mathrm{liter}$ ). Compared to control mitochondria respiring in the presence of glutamate plus malate (Trace A), adding HEX-free ethanol (EtOH; 20 $\mu \mathrm{l}$ in a total volume of $1.605 \mathrm{ml}$; Trace B) had no significant effect on mitochondrial performance. ADP, 500 $\mathrm{nmol}$ of added $\mathrm{Na}_{2}$-adenosine diphosphate. Scale bar denotes equivalent of 100 natom of oxygen (vertical), 1 min of time (horizontal). Increasing concentrations of HEX (0.59 $\mu \mathrm{M}$, Trace C; $5.89 \mu \mathrm{M}$, Trace D; $14.4 \mu \mathrm{M}$, Trace E) produced progressive increases of State 4 respiratory rates, suggesting an uncoupling action.
TABLE 2

EFFECTS OF HEXACHLOROCYCLOPENTADIENE (HEX) ON RESPIRATORY CONTROL AND ADP:O RATIOS OF ISOLATED TROUT HEART MITOCHONDRIA ${ }^{a}$

\begin{tabular}{lcc}
\hline Treatment & RCR $^{b}$ & ADP:O ratio \\
\hline None & $8.7 \pm 0.6$ & $3.04 \pm 0.12$ \\
Ethanol & $7.9 \pm 1.0$ & $3.26 \pm 0.12$ \\
HEX $(\mu \mathrm{M})$ & & \\
0.015 & $8.6 \pm 1.4$ & $3.39 \pm 0.08$ \\
0.059 & $5.7 \pm 1.0^{d}$ & $3.08 \pm 0.68$ \\
0.150 & $5.5 \pm 0.2^{d}$ & $2.93 \pm 0.11$ \\
0.590 & $3.2 \pm 0.7^{d}$ & $2.62 \pm 0.20$ \\
5.9 & $1.7 \pm 0.5^{d . e}$ & N.D. ${ }^{f}$ \\
14.4 & $1.4 \pm 0.4^{d, e}$ & N.D. ${ }^{f}$ \\
\hline
\end{tabular}

${ }^{a}$ Mitochondria were isolated and analyzed as described in text. Values shown are arithmetic $\bar{x} \pm 1$ SEM, obtained from six mitochondrial preparations. For each preparation, each treatment was assayed at least in duplicate. Glutamate plus malate were substrates.

${ }^{b}$ Respiratory control ratio, defined as State 3 respiratory rate/subsequent State 4 respiratory rate.

${ }^{c}$ Ethanol control, $20 \mu \mathrm{l}$ of absolute ethanol in total volume of $1.605 \mathrm{ml}$.

${ }^{d}$ Significantly different ( $p<0.05$, paired $t$ test) from ethanol control value.

"This value is a respiratory control index, defined as State 3 respiratory rate/initial State 4 rate, since there was no return to State 4 after the initial ADP addition.

${ }^{f}$ Not determinable, since these mitochondria did not return to State 4 after initial ADP addition.

respiratory stimulating effect in the intact animal exposed to lethal doses of the diene, and marked increases of basal mitochondrial respiratory rates in vitro, indicative of mitochondrial uncoupling. In the intact organism, the mitochondrial actions of HEX would be manifest as a marked increase of oxygen consumption, which is what we observed. Concomitant with the respiratory stimulation at the expense of ADP phosphorylation would be the impairment of ATP synthesis, another important factor which could lead to death of the organism.

Our data showing a profound effect of HEX on isolated heart mitochondria do not imply necessarily that the primary target of the agent's toxicity in these trout, or in other exposed species, is the heart or other parts of 


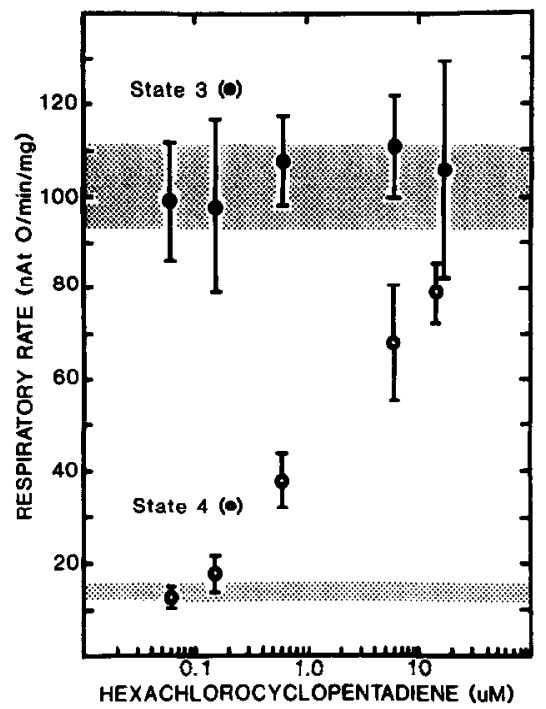

FIG. 5. This figure shows the effects of increasing HEX concentrations on State 3 (solid circles) and State 4 (open circles) respiratory rates. Each data point represents the arithmetic mean of results obtained with six different preparations, each run in duplicate, plus and minus one SEM. The shaded area in the upper portion of the figure denotes the $\bar{x} \pm 1$ SEM of State 3 rates obtained with mitochondria exposed to HEX-free ethanol. The shaded area in the lower portion corresponds to control State 4 rates. At concentrations of $0.59 \mu \mathrm{M}$ and more, HEX significantly increased State 4 respiratory rates, but had no effect on State 3 respiration.

the circulatory system. Results of an ancillary study (Sinhaseni, 1982) of tissue distribution of $\left[{ }^{14} \mathrm{C}\right] \mathrm{HEX}$ in exposed trout showed that the major fraction of recovered HEX ( $40 \%$ of total recovered radioactivity) was found bound to protein of skeletal muscle, which comprised $60 \%$ of the body weight of the fish. Moreover, approximately $30 \%$ of total recovered label was found in liver and in gill structures (about 3\% of total body weight). These results are not unexpected considering the fact that the liver is the primary site of HEX metabolism, and that the gills are the primary route of HEX absorption in this model. However, although brain and heart collectively comprised only $0.2 \%$ of total body weight, they contained a total of $1.5 \%$ of recovered radioactivity $(0.9 \%$ recovery for heart; $0.6 \%$ recovery for brain), suggesting that these or- gans, both of which are highly dependent upon oxidative metabolism for ATP synthesis, accumulated a relatively high fraction of HEX when considered in terms of their relative contribution to total body weight. Thus, it is conceivable that both neurological and cardiovascular dysfunction may be important in the overall toxicity which occurs upon exposure to lethal doses of HEX. Relative tissue distribution of HEX is affected by the route of administration, but the figures cited above (discussed in detail by Sinhaseni, 1982) were obtained by the route of administration used in the current study, and is most relevant to environmental exposure of fish or to inhalational exposure of terrestrial animals.

Although we evaluated the mitochondrial effects of HEX only in terms of oxygen consumption in the presence of a single NADHlinked substrate combination, and did not test the effects of HEX on mitochondrial ATPase or on oligomycin-treated organelles, the experimental evidence is consistent with the hypothesis that HEX can act as a mitochondrial uncoupler, with a molar potency approximately 50 times that generally attributed to 2,4-dinitrophenol. Mitochondrial uncouplers often act by dissipating proton gradients across the mitochondrion, yet HEX itself cannot accept or donate protons to produce such an uncoupling effect. Alternatively some organic molecules can bind to potential uncoupler sites (Hatefi, 1975) to produce a similar phenomenon, and due to the avidity of HEX to covalently bind to proteins (Sinhaseni, 1982), this may serve as an explanation for the effect. Despite the fact that the highest HEX concentration studied $(14.4 \mu \mathrm{M})$ was 35 times that needed to decrease respiratory control by $50 \%$, there was no evidence that HEX acted as an inhibitor of mitochondrial electron transport, a property which is common to some cyclodiene insecticides.

In summary, although HEX is widely distributed throughout the body of exposed animals, our data suggest that a key factor in the toxic actions of HEX is increased oxygen consumption which may be due to uncoupled 
oxidative phosphorylation by the mitochondria.

\section{REFERENCES}

ATAllah, Y. H., WhitaCRE, D. M., AND BUTz, R. G. (1981). Fate of hexachlorocyclopentadiene in the environment. In Toxicology of Halogenated Hydrocarbons. Health and Ecological Effects, pp. 344-355. Pergamon Press, New York.

HATEFI, Y. (1975). Energy conservation and uncoupling in mitochondria. J. Supramol. Struct. 3, 201-213.

Khan, M. A. Q., Sudershan, P., Feroz, M., AND PoDOWSKI, A. A. (1981). Biotransformations of cyclodienes and their photoisomers and hexachlorocyclopentadiene in mammals and fish. In Toxicology of $\mathrm{Ha}$ logenated Hydrocarbons. Health and Ecological Effects, pp. 283-285. Pergamon Press, New York.

KominSKY, J. R., WisSEMAN, C. L., AND MoRSE, D. L. (1980). Hexachlorocyclopentadiene contamination of a municipal wastewater treatment plant. Amer. Ind. Hyg. Assoc. J. 41, 552-556.

LAWRENCE, L. J., AND DOROUGH, H. W. (1981). Retention and fate of inhaled hexachlorocyclopentadiene in the rat. Bull. Environ. Contam. Toxicol. 26, 663-668.

LU, P. Y., Metcalf, R. L., HiRwe, A. S., and Williams, J. W. (1975). Evaluation of environmental distribution and fate of hexachlorocyclopentadiene, chlordane, heptachlor and heptachlor-epoxide in a laboratory model ecosystem. J. Agr. Food Chem. 23, 967-973.

MEHENDALE, H. M. (1977). The chemical reactivity-ab- sorption, retention, metabolism and elimination of hexachlorocyclopentadiene. Environ. Health Perspect. 21, 275-278.

PatTy, F. A. (1963). Cyclic halogenated hydrons. In Industrial Hygiene and Toxicology (D. W. Fassett and D. D. Irish, eds.), Vol. 2, pp. 1360-1361. Interscience, New York.

Prosser, C. L. (1973). Comparative Animal Physiology, 3rd ed., Vol. 1, Saunders, Philadelphia.

Shlafer, M., KIRSH, M. M., LUCChESI, B. R., SlATER, A. D., AND WARREN, S. (1981). Mitochondrial function after global cardiac ischemia and reperfusion: Influences of organelle isolation protocols. Basic Res. Cardiol. 76, 250-266.

SINHASENI, P. (1982). Biological Effects of Hexachlorocyclopentadiene in the Rainbow Trout, Salmo gairdneri, pp. 1-94. Ph.D. thesis, University of Michigan.

SKIDMORE, J. F. (1970). Respiratory and osmoregulation in rainbow trout with gills damaged by zinc sulphate. J. Exp. Biol. 52, 481-494.

Spehar, R. L., Veith, G. D., DeFor, D. L., and BergsTEDT, B. V. (1979). Toxicity and bioaccumulation of hexachlorocyclopentadiene, hexachloronorbornadiene and heptachlorobornene in larval and early juvenile fathead minnows, Pimephales promelas. Bull. Environ. Contam. Toxicol. 21, 576-583.

Trfon, J. F., Clfveland, F. P., AND Cappel, J. (1955). The toxicity of hexachlorocyclopentadiene. AMA Arch. Ind. Health 11, 459-472.

Veith, G. D., DeFoe, D. L., AND Bergstedt, B. V. (1979). Measuring and estimating the bioconcentration factor of chemicals in fish. J. Fish. Res. Bd. Canad. 36, 1040-1048. 